\title{
The M/M/1 Queue with Single Working Vacation Serving at a Slower Rate during the Start-up Period
}

\author{
Chun Xiu, Naishuo Tian \& Yazhen Liu \\ Collage of Science, Yanshan University, Qinhuangdao 066004, China \\ E-mail: xiuchun002@163.com
}

Supported by National Natural Science foundation, China(Grant No. 10671170)

\begin{abstract}
We consider the $\mathrm{M} / \mathrm{M} / 1$ queue with single working vacation serving at a slower rate during the start-up period. In order to save energy and reduce waste, the server works at a slower rate rather than completely stops during a vacation and start-up period. Using quasi birth and death process and matrix-geometric solution method, we obtain the distribution of the nember of customers in the system, the average nember of the customers and the average sojourn time of a customer in the stationary state.
\end{abstract}

Keywords: Start-up period, State transition rate matrix, Quasi birth and death process, Matrix-geometric solution, Single working vacation

\section{Introduction}

Over the past two decades, the vacation queues have been investigated extensively. In a classical vacation queue, a server may completely stop service or do some additional work during a vacation. The vacation queues have been extended to computer networks, communications systems, as well as production management, inventory management and other fields(Doshi B T, 1990).

Recently, a class of semi-vacation policies has been introduced by Servi and Finn. Such a vacation is called a working vacation (WV). The server works at a slower rate rather than completely stops during a vacation. Servi and Finn(Servi, L. D., 2002) studied an M/M/1 queue with multiple working vacations, and obtained the of the number of customers in the system and the of waiting time distribution. Later, Liu, Xu and Tian(2002) gave simple explicit expressions of distribution for the stationary queue length and waiting time which have intuitionistic probability sense. Kin, Choi and Chae, Wu and Takagi(2006) generalized the work of to an M/G/1 queue with multiple working vacations(Wu, D., 2006), Baba investigated a GI/M/1 queue multiple working vacations. Recently, Tian ,Zhao and Wang(2008) study an M/M/1 queue with single working vacation. According to Tian, Zhao and Wang's research£this paper introduced a start-up period. In addition, there is a slow rate of service during the start-up period.

In this paper, we study an $\mathrm{M} / \mathrm{M} / 1$ queue with single working vacation serving at a slower rate in during the start-up period. Firstly, the system is in a closed state, when a customer arrives, leading to a start-up period. After the start-up period, the system becomes a normal service state. Until there are no customers in the queue, it changes into the working vacation state. When the working vacation ends, if there are customers in the queue, the system becomes a normal service state: if there are no customers in the queue, the system is closed. Until a customer arrives, a new cycle begins. In this model, when the number of customers in the system is relatively few, we set a lower service rate. If there are no customers in the system, we close it in order to save energy and reduce waste. Using quasi birth and death process and matrixgeometric solution method, we obtain the distribution of the member of customers in the system, the average member of the customers and the average sojourn time of a customer in the stationary state.

The rest of this paper is organized as follows. In Section 2 we describe the quasi birth and death process model of the system; In Section 3 we obtain the steady- state queue distribution; In Section 4 we obtain the average member of the customers and the average sojourn time of a customer in the stationary state.

\section{Quasi Birth and Death Process}

\subsection{Model Description}

Firstly, the system is in a closed state, when a customer arrives, leading to a start-up period. The start-up period $U$ follows 
an exponential distribution with parameter $\beta$ and the server serves at a slower rate of $\mu_{\beta}$. After the start-up period, the system becomes a normal service state and the server serves at a normal rate of $\mu_{b}$. Until there are no customers in the queue, it changes into the working vacation state. The working vacation time $V$ follows an exponential distribution with parameter $\theta$ and the server serves at a slower rate of $\mu_{v}$. When the working vacation ends, if there are customers in the queue, the server changes service rate from $\mu_{v}$ to $\mu_{b}$ and the system becomes a normal service state; if there are no customers in the queue, the system is closed. Until a customer arrives, a new cycle begins.

We assume that interarrival times, start-up period, service times, and working vacation time are mutually independent. In addition, the service order is first in first out $(F I F O)$.

\subsection{State Transition Rate Matrix}

Let $Q(t)$ be the number of customers in the system at time t and let State variables

$$
J(t)=\left\{\begin{array}{l}
0 \quad \text { the system is in a working vacation period at time } t \\
1 \text { the system is in a start - up period at time } t \\
2 \text { the system is in a regular busy period at time } t
\end{array}\right.
$$

Then $\{Q(t), J(t)\}$ is a process with the state space

$$
\Omega=\{(k, j), k \geq 1, j=0,1,2\} \cup(0,0) \cup(0,1)
$$

Where state $(0,1)$ denotes that the system is in a close-up state; state $(k, 0), k \geq 0$ indicates that the system is in working vacation state and there are $k$ customers in the queue; state $(k, 1), k \geq 1$ indicates that the system is in start-up state and there are $k$ customers in the queue; state $(k, 2), k \geq 1$ indicates that the system is in regular busy period state and there are $k$ customers in the queue.

According to the lexicographical sequence, the state transition rate matrix can be written as

$$
\tilde{Q}=\left[\begin{array}{cccccc}
A_{00} & A_{01} & & & & \\
B_{10} & A & C & & & \\
& B & A & C & & \\
& & B & A & C & \\
& & & \ddots & \ddots & \ddots
\end{array}\right]
$$

where

$$
\begin{gathered}
A_{00}=\left[\begin{array}{cc}
-(\lambda+\theta) & \theta \\
0 & -\lambda
\end{array}\right] \quad A_{01}=\left[\begin{array}{lll}
\lambda & 0 & 0 \\
0 & \lambda & 0
\end{array}\right] \\
A=\left[\begin{array}{ccc}
-\left(\mu_{v}+\theta+\lambda\right) & 0 & \theta \\
0 & -\left(\mu_{\beta}+\beta+\lambda\right) & \beta \\
0 & 0 & -\left(\lambda+\mu_{b}\right)
\end{array}\right] \\
B_{10}=\left[\begin{array}{cc}
\mu_{n} v & 0 \\
0 & \mu_{\beta} \\
\mu_{b} & 0
\end{array}\right] \quad B=\left[\begin{array}{ccc}
\mu_{v} & 0 & 0 \\
0 & \mu_{\beta} & 0 \\
0 & 0 & \mu_{b}
\end{array}\right] \quad C=\left[\begin{array}{lll}
\lambda & 0 & 0 \\
0 & \lambda & 0 \\
0 & 0 & \lambda
\end{array}\right]
\end{gathered}
$$

The structure of $\tilde{Q}$ indicates that $\{Q(t), J(t)\}$ is a quasi birth and death process $(Q B D)$, see Neuts. (1981).

Theorem 1. If $\rho=\frac{\lambda}{\mu_{b}}<1$, the matrix equation

$$
R^{2} B+R A+C=0
$$

has the minimal non-negative solution

$$
R=\left[\begin{array}{ccc}
r & 0 \frac{\theta r}{\lambda+(1-r-\rho) \mu_{b}} & \\
0 & \varepsilon & \frac{\beta \varepsilon}{\lambda+(1-\varepsilon-\rho) \mu_{b}} \\
0 & 0 & \rho
\end{array}\right]
$$

Where 


$$
\begin{gathered}
\rho=\frac{\lambda}{\mu_{b}} \quad r=\frac{\lambda+\theta+\mu_{v}-\sqrt{\left(\lambda+\theta+\mu_{v}\right)^{2}-4 \lambda \mu_{v}}}{2 \mu_{v}} \\
\varepsilon=\frac{\lambda+\beta+\mu_{\beta}-\sqrt{\left(\lambda+\beta+\mu_{\beta}\right)^{2}-4 \lambda \mu_{\beta}}}{2 \mu_{\beta}}
\end{gathered}
$$

Proof. Because $A, B, C$ are all upper-triangular, we can assume that $R$ has the same structure as

$$
R=\left[\begin{array}{ccc}
r_{11} & r_{12} & r_{13} \\
0 & r_{22} & r_{23} \\
0 & 0 & r_{33}
\end{array}\right]
$$

Substituting $R^{2}$ and $R$ into equation (1), we get

$$
\begin{gathered}
r_{11}=\frac{\lambda+\theta+\mu_{v}-\sqrt{\left(\lambda+\theta+\mu_{v}\right)^{2}-4 \lambda \mu_{v}}}{2 \mu_{v}} \quad r_{22}=\frac{\lambda+\beta+\mu_{\beta}-\sqrt{\left(\lambda+\beta+\mu_{\beta}\right)^{2}-4 \lambda \mu_{\beta}}}{2 \mu_{\beta}} \\
r_{12}=0 \quad r_{23}=\frac{r_{22} \beta}{\lambda+\left(1-r_{22}-r_{33}\right)} \\
r_{13}=\frac{r_{11} \theta}{\lambda+\left(1-r_{11}-r_{33}\right)} \quad r_{33}=\frac{\lambda}{\mu_{b}}
\end{gathered}
$$

To obtain the minimal non-negative solution of (1), taking $r_{11}=r$ (the other root is greater than 1), taking $r_{22}=\varepsilon$ (the other root is greater than 1), taking $r_{33}=\rho$ (the other root is $r_{33}=1$ ). Using elementary method, we can prove that $0<r<1,0<\varepsilon<1$. Substituting $r, \varepsilon, \rho$ into equation, we get $r_{12}, r_{13}, r_{23}$.

Because $r$ satisfies the following equation

$$
\mu_{\nu} r^{2}-\left(\lambda+\theta+\mu_{v}\right)+\lambda=0
$$

equivalently, we have

$$
\frac{\theta}{1-r}+\mu_{v}=\frac{\lambda}{r}
$$

Similarly, we can have

$$
\frac{\beta}{1-\varepsilon}+\mu_{\beta}=\frac{\lambda}{\varepsilon}
$$

Theorem 2. The $Q B D$ process $\{Q(t), J(t)\}$ is positive recurrent if and only if $\rho<1$. Proof. Based on the theorem of Neuts, the $Q B D$ process $\{Q(t), J(t)\}$ is positive recurrent if and only if the spectral radius $S P(R)$ of the rate matrix $R$ is less than 1 , and set of equations $\left(x_{0}, x_{1}, x_{2}, x_{3}, x_{4}\right) B[R]=0$ has positive solution, where

$$
B[R]=\left[\begin{array}{cc}
A_{00} & A_{01} \\
B_{10} & R B+A
\end{array}\right]=\left[\begin{array}{ccccc}
-(\lambda+\theta) & \theta & \lambda & 0 & 0 \\
0 & \lambda & 0 & \lambda & 0 \\
\mu_{v} & 0 & -\frac{\lambda}{r} & 0 & \frac{\lambda}{r}-\mu_{v} \\
0 & \mu_{\beta} & 0 & -\frac{\lambda}{\varepsilon} & \frac{\lambda}{\varepsilon}-\mu_{\beta} \\
\mu_{b} & 0 & 0 & 0 & -\mu_{b}
\end{array}\right]
$$

$B[R]$ is an irreducible and a periodic generator with finite state. Therefore, $\left(x_{0}, x_{1}, x_{2}, x_{3}, x_{4}\right) B[R]=0$ has positive solution. Thus, process $\{Q(t), J(t)\}$ is positive recurrent if and only if

$$
S P(R)=\max (r, \varepsilon, \rho)<1
$$

where $0<r<1,0<\varepsilon<1$, the above relation means that $\rho<1$.

\section{Steady- state Queue Length Distribution}

If $\rho<1, \mu_{v}<\mu_{b}, \mu_{\beta}<\mu_{b}$, let $(Q, J)$ be the stationary limit of the $Q B D$ process. Let

$$
\begin{gathered}
\pi_{k j}=P\{Q=k, J=j\},(k, j) \in \Omega \\
\left(\pi_{k 0}, \pi_{k 1}, \pi_{k 2}\right)=\pi_{k}, k \geq 1
\end{gathered}
$$


Theorem 3. If $\rho<1, \mu_{v}<\mu_{b}, \mu_{\beta}<\mu_{b}$, the joint probability distribution of $(Q, J)$ is

$$
\begin{gathered}
\pi_{k 0}=r^{k} \pi_{00} \quad \pi_{k 1}=\frac{\theta(1-\varepsilon)}{\beta} \varepsilon^{k-1} \pi_{00} \\
\pi_{k 2}=\left[(\delta+\varphi) \sum_{j=0}^{k-1} r^{j} \rho^{k-1-j}+(\gamma-\delta-\varphi) \rho^{k-1}\right] \pi_{00}
\end{gathered}
$$

where

$$
\begin{gathered}
\delta=\frac{\theta r}{\lambda+(1-r-\rho) \mu_{b}}, \quad \gamma=\frac{\lambda+\theta-r \mu_{v}}{\mu_{b}}, \quad \varphi=\frac{\theta(1-\varepsilon)}{\lambda+(1-\varepsilon-\rho) \mu_{b}} \\
\pi_{00}=\frac{(1-r)(1-\rho)(1-\varepsilon)}{\left(1+\frac{\theta}{\beta}\right)(1-r)(1-\rho)(1-\varepsilon)+\frac{\theta}{\varepsilon}(1-r)(1-\rho)+r(1-\varepsilon)(1-\rho)+(\delta+\phi)(1-\varepsilon)}+(\gamma-\delta-\varphi)(1-\varepsilon)(1-r)
\end{gathered}
$$

Proof. With the matrix-geometric solution method, we have

$$
\pi_{k}=\left(\pi_{k 0}, \pi_{k 1}, \pi_{k 2}\right)=\left(\pi_{10}, \pi_{11}, \pi_{12}\right) R^{k-1}=0
$$

and $\pi_{0}, \pi_{1}$ satisfy the set of equations

$$
\left[\begin{array}{lllll}
\pi_{00} & \pi_{01} & \pi_{10} & \pi_{11} & \pi_{12}
\end{array}\right] B[R]=0
$$

Substituting $B[R]$ in (3) into the equation, we get

$$
\begin{array}{cc}
\pi_{01}=\frac{\theta(1-\varepsilon)}{\varepsilon \beta} \pi_{00} & \pi_{10}=r \pi_{00} \\
\pi_{11}=\operatorname{frac} \theta(1-\varepsilon) \beta \pi_{00} & \pi_{12}=\frac{\lambda+\theta-r \mu_{v}}{\mu_{b}} \pi_{00}
\end{array}
$$

note that

$$
R^{k}=\left[\begin{array}{ccc}
r^{k} & 0 & \frac{\theta r}{\lambda+(1-r-\rho) \mu_{b}} \sum_{j=0}^{k-1} r^{j} \rho^{k-1-j} \\
0 & \varepsilon^{k} & \frac{\beta \varepsilon}{\lambda+(1-\varepsilon-\rho) \mu_{b}} \sum_{j=0}^{k-1} r^{j} \rho^{k-1-j} \\
0 & 0 & \rho^{k}
\end{array}\right], k \geq 1
$$

Substituting $\left(\pi_{10}, \pi_{11}, \pi_{12}\right)$ and $R^{k-1}$ into (5), we obtain (4). Finally, $\pi_{00}$ can be determined by the normalization condition. With (4), the probabilities of the server in various state are as follows, respectively

$$
\begin{gathered}
P\{\text { the server is in close - up period }\}=\pi_{01}=\frac{\theta(1-\varepsilon)}{\varepsilon \beta} \pi_{00} \\
P\{\text { the server is in start - up period }\}=P\{J=1\}=\sum_{k=1}^{\infty} \pi_{k 1}=\frac{\theta}{\beta} \pi_{00} \\
P\{\text { the server is in working vacation period }\}=P\{J=0\}=\sum_{k=1}^{\infty} \pi_{k 0}=\frac{1}{1-r} \pi_{00} \\
P\{\text { the server is in regular busy period }\}=P\{J=2\}=\sum_{k=1}^{\infty} \pi_{k 2}=\left[(\delta+\varphi) \frac{1}{1-r} \frac{1}{1-\rho}+\frac{\gamma-\delta-\varphi}{1-\rho}\right] \pi_{00}
\end{gathered}
$$

\section{The Average of the Queue Length and the Sojourn Time in Steady State}

Theorem 4. If $\rho<1, \mu_{v}<\mu_{b}, \mu_{\beta}<\mu_{b}$, the average of the queue length in steady state

$$
E(L)=\left[\frac{1}{(1-r)^{2}}+\frac{\theta}{1-\varepsilon}+\frac{(\delta+\varphi)(1-r \rho)}{(1-\rho)^{2}(1-r)^{2}}+\frac{\gamma-\delta-\varphi}{(1-\rho)^{2}}\right] \pi_{00}
$$

the average of the sojourn time in steady state

$$
E(W)=\frac{1}{\lambda}\left[\frac{1}{(1-r)^{2}}+\frac{\theta}{1-\varepsilon}+\frac{(\delta+\varphi)(1-r \rho)}{(1-\rho)^{2}(1-r)^{2}}+\frac{\gamma-\delta-\varphi}{(1-\rho)^{2}}\right] \pi_{00}
$$


Proof. With (4), the probability generating function of $Q$ cab be written as

$$
Q(z)=\sum_{k=0}^{\infty}\left(\pi_{k 0}+\pi_{k 1}+\pi_{k 2}\right) z^{k}=\left[1+\frac{\theta(1-\varepsilon)}{\varepsilon \beta}+\frac{r z}{1-r z}+\theta(1-\varepsilon) \frac{z}{1-\varepsilon z}+(\delta+\varphi) \frac{z}{1-\rho z} \frac{1}{1-r z}+(\gamma-\delta-\varepsilon) \frac{z}{1-\rho z}\right] \pi_{00}
$$

Therefore

$$
E(L)=Q^{\prime}(z) \mid z=1=\left[\frac{1}{(1-r)^{2}}+\frac{\theta}{1-\varepsilon}+\frac{(\delta+\varphi)(1-r \rho)}{(1-\rho)^{2}(1-r)^{2}}+\frac{\gamma-\delta-\varphi}{(1-\rho)^{2}}\right] \pi_{00}
$$

If the $P G F$ of $W$ is $W(s)$, the relationship between the $P G F$ of $Q$ and $W$ is

$$
Q(z)=W(\bar{\lambda}+\lambda z)
$$

therefore

$$
E(W)=W^{\prime}(s)\left|s=1=\frac{1}{\lambda} Q^{\prime}\left(\frac{s-\bar{\lambda}}{\lambda}\right)\right| s=1
$$

\section{References}

Doshi B T. (1990). Single server queueswith vacations in: H. Takagi (Ed), Stochastic Analysis of the coputer and communication Systems. North2Holland Elsevier, Amsterdam, 217-264.

Liu, W., Xu, X. and Tian, N. (2002). Some results on the M/M/1 queue with working vacations. Oper. Res.Letters, Vol. 50, pp. 41-52.

Neuts, M. (1981). Matrix-Geometric Solution in Stochastic models, Johns Hopking University Press, Baltimore.

Servi, L. D. and Finn, S. G. (2002). M/M/1 queue with working vacations(M/M/1/WV). Performance Evaluation, Vol. 50, pp. 41-52.

Tian, Zhao and Wang. (2008). The M/M/1 queue with single working vacation. International Journal of Information and Management Science, Vol. 19, No. 4, pp. 621-634.

Wu, D. and Takagi, H. (2006). M/G/1 queue with multiple working vacation. Performance Evaluation, Vol. 63, No. 7, pp. 654-681. 